\title{
The Influence of the Cloud Shell on Tracer Budget Measurements of LES Cloud Entrainment
}

\author{
Jordan T. DAWE AND PHILIP H. AUSTIN \\ Department of Earth and Ocean Sciences, University of British Columbia, Vancouver, British Columbia, Canada
}

(Manuscript received 30 August 2010, in final form 20 July 2011)

\begin{abstract}
Direct measurements of rates of entrainment into and detrainment from cumulus cloud cores obtained from LES model cloud fields produce values twice as large as those produced from tracer budget calculations. This difference can be explained by two effects: the presence of a shell of air around the cloud cores that is moister than the mean environment and air at the edge of the cloud core that is drier than the mean core, and the tendency for the mean tracer values of the entrained fluid to be greater than the mean tracer values of the cloud shell. Preferential entrainment of shell air that is moving upward faster than the mean shell creates strong vertical momentum fluxes into the cumulus cloud core, thereby making the assumption that cumulus cloud cores entrain fluid with zero vertical momentum incorrect. Variability in the properties of the moist cloud shell has strong impacts on entrainment values inferred from tracer budget calculations. These results indicate that the dynamics of the cloud shell should be included in parameterization of cumulus clouds used in general circulation models.
\end{abstract}

\section{Introduction}

The rate at which air is entrained into and detrained from cumulus clouds affects cloud properties, cloud-top height, and vertical transports of heat and moisture. Proper simulation of cumulus subgrid-scale fluxes in general circulation models (GCMs) depends on the accurate parameterization of entrainment of environmental properties into the clouds and detrainment of cloud properties into the environment (Bechtold et al. 2008; de Rooy and Siebesma 2010).

Entrainment and detrainment may be defined mathematically as

$$
\begin{aligned}
& E=-\frac{1}{A} \oint_{\hat{\mathbf{n}} \cdot\left(\mathbf{u}-\mathbf{u}_{i}\right)<0} \rho \hat{\mathbf{n}} \cdot\left(\mathbf{u}-\mathbf{u}_{i}\right) d l, \\
& D=\frac{1}{A} \oint_{\hat{\mathbf{n}} \cdot\left(\mathbf{u}-\mathbf{u}_{i}\right)>0} \rho \hat{\mathbf{n}} \cdot\left(\mathbf{u}-\mathbf{u}_{i}\right) d l,
\end{aligned}
$$

where $E$ and $D$ are the entrainment and detrainment rates $\left(\mathrm{kg} \mathrm{m}^{-3} \mathrm{~s}^{-1}\right), \rho$ is the density of air $\left(\mathrm{kg} \mathrm{m}^{-3} \mathrm{~s}^{-1}\right)$,

Corresponding author address: Jordan T. Dawe, Department of Earth and Ocean Sciences, University of British Columbia, 6339 Stores Road, Vancouver, BC V6T 1Z4, Canada.

E-mail: jdawe@eos.ubc.ca $\mathbf{u}$ is the velocity of the air $\left(\mathrm{m} \mathrm{s}^{-1}\right), \mathbf{u}_{i}$ is the velocity of the cloud surface $\left(\mathrm{m} \mathrm{s}^{-1}\right), A$ is the area of the cloud $\left(\mathrm{m}^{2}\right), \hat{\mathbf{n}}$ is a unit vector directed out the cloud surface, and the path integral is taken around the cloud surface at a constant vertical level (Siebesma 1998). Entrainment and detrainment are thus caused by differences between the motion of the cloud surface and the motion of the air. This includes not just mixing processes, but also adiabatic processes such as condensation of water vapor at cloud base. Many parameterizations use the cloud core as the region over which to consider entrainment and detrainment, defined as regions having condensed liquid water, positive buoyancy, and upward vertical velocity. In this case, the motion of the cloud core surface is simply substituted for the motion of the cloud surface in Eqs. (1).

Entrainment and detrainment rates impact GCM parameterizations in several ways. First, profiles of cloud vertical mass flux are usually calculated from parameterized entrainment values using the continuity equation for a simple entraining plume to represent an ensemble of cumulus clouds:

$$
\rho \frac{\partial a}{\partial t}+\frac{\partial M_{\text {core }}}{\partial z}=E-D .
$$

Here $a$ is the fractional cloud core area and $M_{\text {core }}$ is vertical cloud core mass flux $\left(\mathrm{kg} \mathrm{m}^{-2} \mathrm{~s}^{-1}\right)$. The level 
where the mass flux profile goes to zero then defines the location of the cloud ensemble top. This mass flux profile is combined with the entrainment rate of environmental air into the cloud and the detrainment rate of cloud air into the environment to generate vertical profiles of cloud water vapor, condensate, and temperature, and these profiles are then used to calculate the moistening of the environment by detrainment of cloud fluid (Tiedtke 1989; Kain and Fritsch 1990). Precipitation rates are also generated from the mass flux and tracer profiles produced from the entrainment and detrainment profiles. This wide variety of effects make entrainment rate one of the strongest controls on the climate sensitivity of GCMs (Stainforth et al. 2005; Rougier et al. 2009).

Large-eddy simulation (LES) is the primary tool used to study cloud entrainment. LES mass entrainment and detrainment rates are typically obtained using budgets of conserved tracer variables to infer the amount of fluid exchange between the cloud ensemble and the surrounding air. Siebesma and Cuijpers (1995) derive the following equations for entrainment and detrainment of mass from the ensemble of cloud core plumes:

$$
\begin{aligned}
E_{\phi}\left(\phi_{\text {core }}-\phi_{\text {env }}\right)= & -M_{\text {core }} \frac{\partial \phi_{\text {core }}}{\partial z}-\frac{\partial \rho a \overline{w^{\prime} \phi^{\prime}} \text { core }}{\partial z} \\
& -\rho a \frac{\partial \phi_{\text {core }}}{\partial t}+a \rho\left(\frac{\partial \bar{\phi}}{\partial t}\right)_{\text {forcing }}
\end{aligned}
$$

and

$$
\begin{aligned}
D_{\phi}\left(\phi_{\text {core }}-\phi_{\text {env }}\right)= & -M_{\text {core }} \frac{\partial \phi_{\text {env }}}{\partial z}+\frac{\partial \rho(1-a) \overline{w^{\prime} \phi^{\prime}} \text { env }}{\partial z} \\
& +\rho(1-a) \frac{\partial \phi_{\text {env }}}{\partial t} \\
& -\rho(1-a)\left(\frac{\partial \bar{\phi}}{\partial t}\right)_{\text {forcing }}
\end{aligned}
$$

where $\phi$ (units denoted by $[\phi]$ ) represents any conserved tracer, such as the total specific humidity $q_{t}$ (kilograms of water per kilogram of moist air) or the liquid-water moist static energy $h\left(\mathrm{~J} \mathrm{~kg}^{-1}\right) ; w$ is vertical velocity $\left(\mathrm{m} \mathrm{s}^{-1}\right)$; the sub- and superscripts "env" and "core" denote horizontally averaged values conditionally sampled in the cloud environment and core, respectively; "forcing" refers to tracer sources and sinks, such as radiation or large-scale subsidence, not included in the other terms; primed values represent anomalies relative to the horizontal mean; overbars represent horizontal averaging; and $E_{\phi}(z)$ and $D_{\phi}(z)$ are the total mass entrainment rate into and detrainment rate from the cloud core inferred from the tracer budget $\left(\mathrm{kg} \mathrm{s}^{-1} \mathrm{~m}^{-3}\right)$. Under this budget formulation, $E_{\phi}(z)$ and $D_{\phi}(z)$ consist of all horizontal mass exchanges between clouds and their environment, including advective processes, diffusive processes, and thermodynamic phase changes. We shall refer to values calculated by this method as "tracer budget" entrainment and detrainment. For convenience, the various tracer and entrainment/detrainment rate subscripts used below are summarized in the appendix.

Alternatively, entrainment and detrainment of mass can be calculated directly from the LES velocity and tracer fields. Romps (2010) recently presented a technique to measure local (grid scale) mass entrainment rate $e(x, y, z)$ and detrainment rate $d(x, y, z)$. His Eq. (2) is

$$
e-d=\frac{\partial}{\partial t}(\mathcal{A} \rho)+\nabla \cdot(\rho \mathbf{u} \mathcal{A})
$$

Here $\mathcal{A}$ is the "activity" of the fluid, where $\mathcal{A}$ is 1 at cloud core points and 0 otherwise. The values of $e-d$ are averaged over the time that a grid cell experiences mass fluxes between an active and an inactive point; positive $e-d$ values are considered to be purely $e$ and negative values, $d$. Summing these point measurements horizontally gives $E_{d}(z)$ and $D_{d}(z)$, the total mass entrained into and detrained from the cloud core field $\left(\mathrm{kg} \mathrm{s}^{-1} \mathrm{~m}^{-3}\right)$, where the subscript $d$ indicates that these quantities were calculated directly from the model velocity and tracer fields. We shall refer to entrainment and detrainment values calculated by this method as "direct" entrainment and detrainment.

Romps found that such direct calculation of the entrainment and detrainment mass fluxes produced values roughly twice as large as tracer budget calculations. Romps attributed this difference to the tracer budget calculation assumption that fluid exchanged between clouds and environment has the mean properties of the cloud ensemble or environment at that level, respectively. Studies of the dense, descending shell of moist air that forms around trade wind cumulus clouds (Jonas 1990; Rodts et al. 2003; Heus and Jonker 2008; Jonker et al. 2008; Heus et al. 2009; Wang and Geerts 2010) suggest that the cloud shell properties are quite different than the core or environment properties, bolstering Romps' hypothesis. Since fluid exchanges between clouds and environment must pass through this shell, it is likely that it plays an important role in entrainment and detrainment dynamics.

Below we examine the sources of the discrepancy in entrainment and detrainment values calculated via tracer budgets and directly using Eq. (4). We show that the discrepancy is explained by two effects: the presence of the shell of moist air around the cloud cores and drier air at 
the edge of the cloud core, and preferential entrainment of shell air with higher average humidity and upward velocity than the mean shell properties, which enhances tracer fluxes between the clouds and the environment. We derive a relation to transform the direct mass entrainment and detrainment rates into tracer budget values suitable for use in one-dimensional simple entraining plume cloud parameterizations, and then use these transformed fluxes to evaluate the impact of the shell on tracer budget entrainment and detrainment rates of specific humidity and vertical velocity. Finally, we examine the dynamics that drives the preferential entrainment of air with higher than average specific humidity and vertical velocity.

\section{Model description}

All LES calculations in this paper were made using the System for Atmospheric Modeling (SAM; Khairoutdinov and Randall 2003). Two model runs were performed, configured as standard Global Energy and Water Cycle Experiment (GEWEX) Cloud System Studies (GCSS; Randall et al. 2003) experiments: a Barbados Oceanographic and Meteorological Experiment (BOMEX; Siebesma et al. 2003) run and an Atmospheric Radiation Measurement Study (ARM; Brown et al. 2002) run. The BOMEX run was performed on a $6.4 \mathrm{~km} \times 6.4 \mathrm{~km}$ horizontal $\times 3.2 \mathrm{~km}$ vertical domain with 25 -m grid size in all directions for $6 \mathrm{~h}$, and the first $3 \mathrm{~h}$ of simulation were discarded. The ARM run was performed on a $7.68 \mathrm{~km} \times$ $7.68 \mathrm{~km} \times 4.5 \mathrm{~km}$ domain with $30-\mathrm{m}$ grid size. Precipitation was disabled in both runs.

We have implemented the direct entrainment calculation scheme of Romps (2010) in SAM, allowing us to calculate the mass of air entrained into and detrained from cloud core directly from model $\rho$, $\mathbf{u}$, and $\mathcal{A}$. Romps [2010, his Eq. (4)] also presents a method for calculating local entrainment and detrainment rates for any model variable in the same framework as our Eq. (4) but neglects forcing terms. These terms are significant for quantities such as vertical momentum, so we modify Romps' equation to include their effects:

$$
e \phi-d \phi=\frac{\partial}{\partial t}(\phi \mathcal{A} \rho)+\nabla \cdot(\phi \rho \mathbf{u} \mathcal{A})-\rho \mathcal{A} S_{\phi},
$$

where $S_{\phi}$ is any nonadvective and nondiffusive source or sink term for $\phi$, such as precipitation for $q_{t}$ or pressure gradient for $w$ (units of $[\phi] \mathrm{s}^{-1}$ ). Diffusion is excluded from $S_{\phi}$ as it is part of the entrainment/detrainment process and so is included in the $e \phi-d \phi$ term of the equation. The inclusion of these source/sink terms allows us to expand the definition of $\phi$ to include nonconserved fluid properties.
As with Eq. (4), the local $(e \phi)(x, y, z)$ and $(d \phi)(x, y, z)$ must be horizontally summed to give the total entrainment into or detrainment out of the cloud ensemble for any fluid property, but since $\phi$ can be negative for properties such as vertical velocity, it is possible for entrainment to reduce and for detrainment to increase the various properties of the cloud core. To accommodate this effect, if the average value of $\phi$ is positive over the time that a grid cell experiences mass fluxes between an active and an inactive grid cell, then positive $e \phi-$ $d \phi$ values are considered to be purely $e \phi$ and negative values, $d \phi$. However, if the average of $\phi$ is negative, then positive $e \phi-d \phi$ values are considered to be purely $(d \phi)(x, y, z)$ and negative values, $(e \phi)(x, y, z)$.

The obvious way to calculate the average value of $\phi$ is to perform a flux-weighted calculation, so that $\phi=$ $(e \phi-d \phi) /(e-d)$. However, doing so for positive definite quantities, such as $q_{t}$, sometimes results in negative $\phi$. To see the reason for this, consider a situation in which $e-d$ integrated over the period that a grid cell is active is found to be slightly bigger than zero for a grid cell. The Romps algorithm would assign $e$ to a small value and $d$ to be zero, but this is only one of many equally valid choices; as long as $e \approx d$, the net flux measured by the algorithm would be satisfied. At the same time, $(e q)_{d}-(d q)_{d}\left\{\left[(e \phi)_{d}-(d \phi)_{d}\right]\right.$ using $q_{t}$ for $\left.\phi\right\}$ is found to be negative, presumably due to $e$ and $d$ having similar magnitudes while the detraining air has higher humidity than the entraining air. In this case, $\left[(e q)_{d}-(d q)_{d}\right] /(e-d)$ will be negative, even though $q_{t}$ is always positive. To avoid this problem, we calculate $\phi$ as a simple time average for the purpose of determining if $e \phi-d \phi$ is assigned to $e \phi$ or to $d \phi$. Horizontal summation of $(e \phi)$ and $(d \phi)$ then gives $(E \phi)_{d}(z)$ and $(D \phi)_{d}(z)$, the total entrainment and detrainment of a property for the cloud ensemble (units of $[\phi] \mathrm{kg} \mathrm{s}^{-1} \mathrm{~m}^{-3}$ ) calculated directly from the model velocity and property fields.

\section{Relationship between direct and tracer budget entrainment}

Romps (2010) established that the direct estimate of mass entrainment and detrainment yields values roughly twice the size of those calculated via conserved tracer budgets. Furthermore, examination of the ratios of the mass entrainment and detrainment calculated via a total specific water budget $\left(E_{q}, D_{q}\right)$ to the directly calculated values $\left(E_{d}, D_{d}\right)$ over the diurnal cycle of an ARM LES reveals significant changes over the course of the day (Fig. 1). Thus, the direct and tracer budget measurements of $E$ and $D$ are not only significantly different but also have differing dynamics, which may need to be accounted for in large-scale parameterizations of cloud 

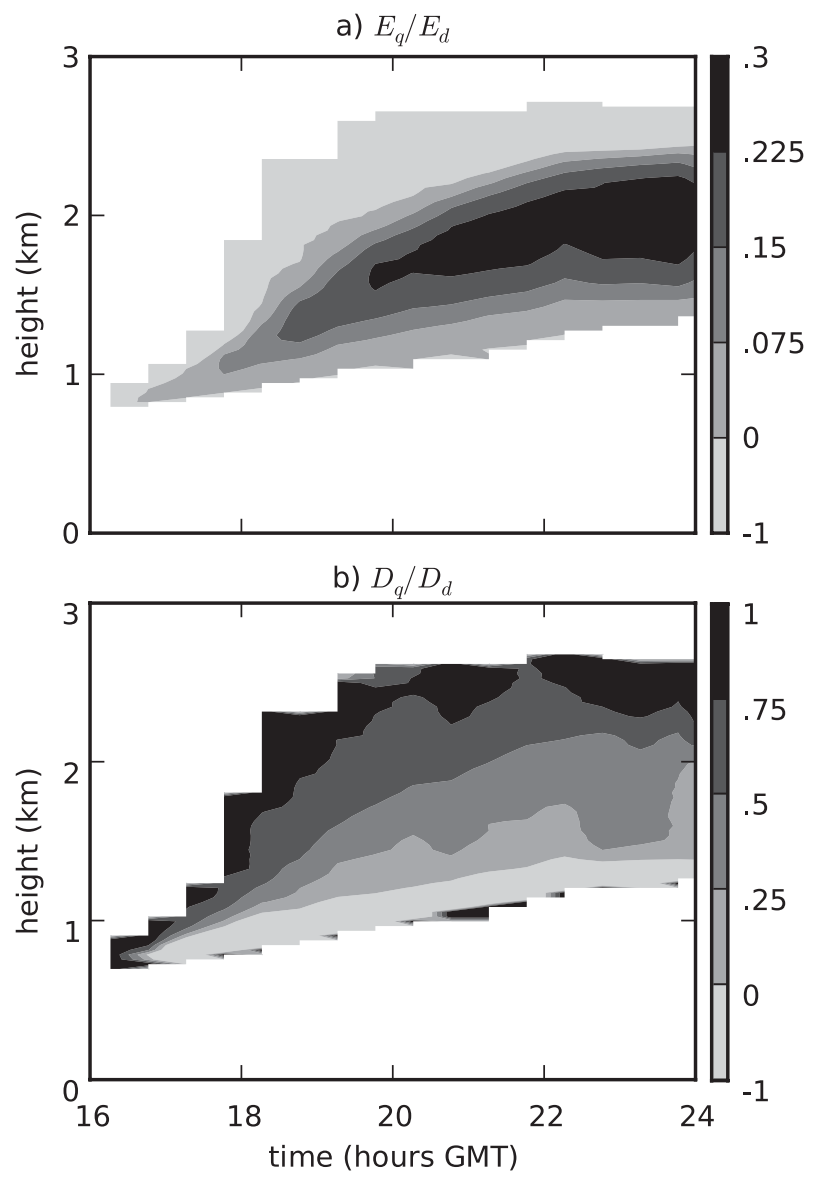

FIG. 1. Ratio of the total specific water tracer budget (a) entrainment and (b) detrainment values to the directly calculated values over the duration of the ARM model run.

entrainment and detrainment. In this section we examine the sources of disagreement between direct and tracer budget estimates of mass entrainment into and detrainment from the cloud core.

\section{a. E and D cloud shell correction}

Romps attributed the differences between $\left(E_{\phi}, D_{\phi}\right)$ and $\left(E_{d}, D_{d}\right)$ to the assumption made by Siebesma and Cuijpers (1995) that fluid being entrained or detrained has the properties of the mean environment or cloud core, respectively. If we examine the horizontal mean specific humidity of the fluid at the "cloud core edge" (cloud core model grid cells that are nearest-neighbor adjacent to noncore cells), which presumably is the fluid being detrained, we see that it is indeed drier than the mean core (Fig. 2a). Similarly, the fluid available for entrainment in the "cloud core shell" (noncore model grid cells that are nearest-neighbor adjacent to core cells) is moister than the mean environment.
Budget equations that explicitly distinguish between the mean cloud core and environment properties and the properties of the entraining and detraining fluid allow us to transform $\left(E_{d}, D_{d}\right)$ values into corresponding tracer budget values, and back again. We start our derivation with the observation that both the tracer budget and direct values of $E$ and $D$ are consistent with the continuity equation [Eqs. (2) and (4)]. This implies that

$$
E_{\phi}-D_{\phi}=E_{d}-D_{d}
$$

Similarly, the entrainment and detrainment rates of fluid properties must be consistent with the total property budget, giving us

$$
E_{\phi} \phi_{\mathrm{env}}-D_{\phi} \phi_{\mathrm{core}}=E_{d} \phi_{E}-D_{d} \phi_{D}
$$

where $\phi_{E}$ and $\phi_{D}$ represent the $\phi$ of the fluid being entrained or detrained, respectively. Combining these equations and solving for $E_{\phi}$ and $D_{\phi}$ in turn results in

$$
\begin{gathered}
E_{\phi, T}=E_{d}-\left(E_{d} \frac{\phi_{E}-\phi_{\mathrm{env}}}{\phi_{\mathrm{core}}-\phi_{\mathrm{env}}}+D_{d} \frac{\phi_{\mathrm{core}}-\phi_{D}}{\phi_{\mathrm{core}}-\phi_{\mathrm{env}}}\right), \\
D_{\phi, T}=D_{d}-\left(E_{d} \frac{\phi_{E}-\phi_{\mathrm{env}}}{\phi_{\mathrm{core}}-\phi_{\mathrm{env}}}+D_{d} \frac{\phi_{\text {core }}-\phi_{D}}{\phi_{\text {core }}-\phi_{\text {env }}}\right) .
\end{gathered}
$$

Here the subscript $T$ indicates that these $E_{\phi}$ and $D_{\phi}$ tracer budget values have been calculated by transformation of direct values, not via Eqs. (3). Ideally these transformed values should be identical to the tracer budget values, but numerical errors may result in differences between the two calculation methods and so we use the subscript $T$ to make clear the method used to calculate each entrainment and detrainment value. The bracketed terms represent the bias introduced by assuming that entrained/detrained air has the properties of the mean environment and core. Thus, to convert from $\left(E_{d}, D_{d}\right)$ to $\left(E_{\phi, T}, D_{\phi, T}\right)$, both $E_{d}$ and $D_{d}$ must be reduced by $E_{d} A+D_{d} B$, where $A=\left(\phi_{E}-\phi_{\text {env }}\right) /\left(\phi_{\text {core }}-\right.$ $\left.\phi_{\text {env }}\right)$ and $B=\left(\phi_{\text {core }}-\phi_{D}\right) /\left(\phi_{\text {core }}-\phi_{\text {env }}\right)$.

Note that rearrangement of $A$ gives $\phi_{E}=A \phi_{\text {core }}+(1-$ A) $\phi_{\text {env }}$, meaning that $A$ can be thought of as the fraction of mean core air in a mixture of mean core and mean environment air needed to produce the properties of the entrained fluid. Similarly, $\phi_{D}=B \phi_{\text {env }}+(1-B) \phi_{\text {core }}$ and $B$ can be thought of as the fraction of mean environment air in a mixture of mean core and mean environment air needed to produce the properties of the detrained fluid. 

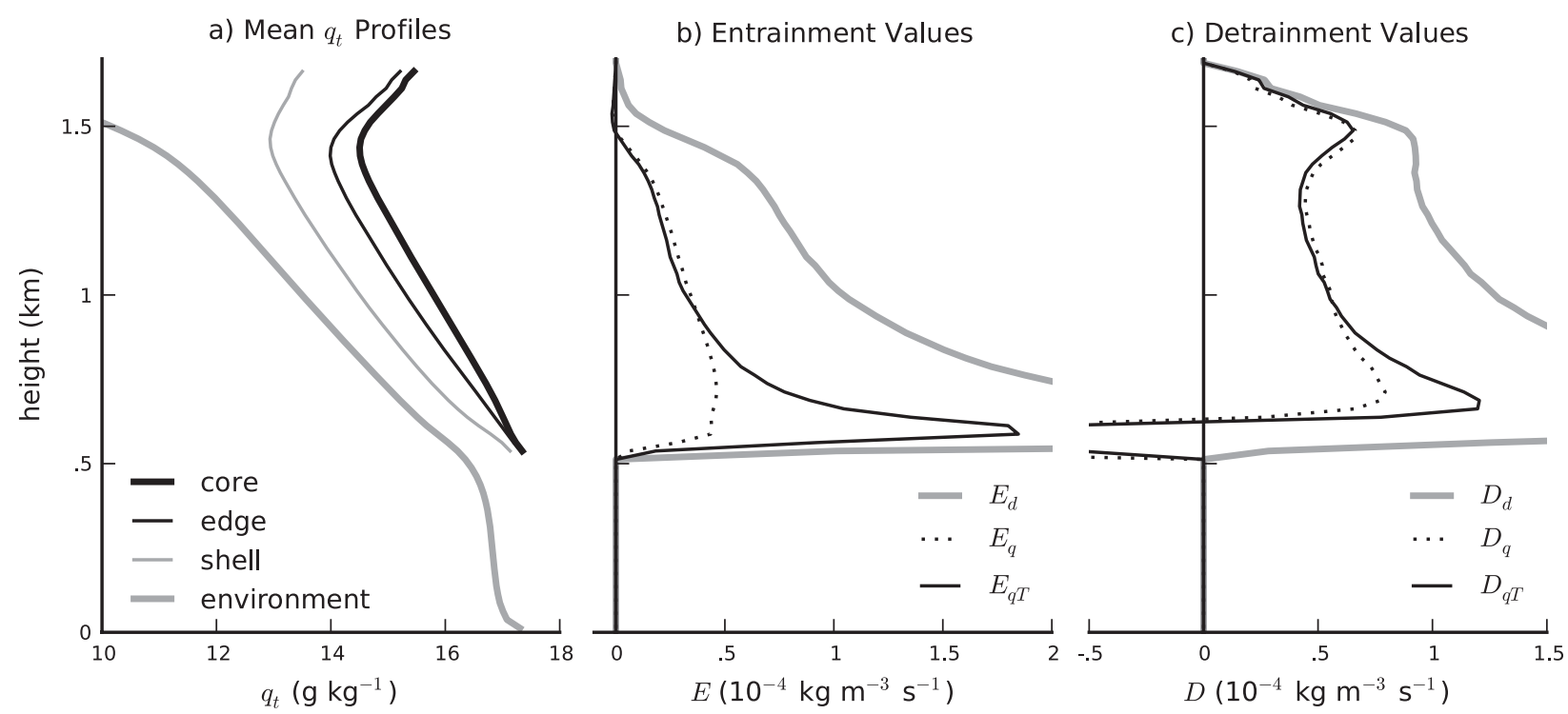

FIG. 2. Result of transforming direct entrainment values into equivalent tracer budget values using mean cloud core shell and edge properties from the BOMEX simulation. (a) Mean profiles of the total specific humidity in the cloud core (thick black line), cloud core edge (thin black line), cloud core shell (thin gray line), and cloud core environment (thick gray line). These $q_{t}$ values are used to transform directly calculated values of (b) entrainment and (c) detrainment (gray line) into equivalent tracer budget values (black line). The tracer budget entrainment and detrainment are shown for comparison (dotted lines).

Alternatively, we can solve for $E_{d}$ and $D_{d}$, arriving at

$$
\begin{aligned}
& E_{d, T}=E_{\phi}+\left(E_{\phi} \frac{\phi_{E}-\phi_{\mathrm{env}}}{\phi_{D}-\phi_{E}}+D_{\phi} \frac{\phi_{\mathrm{core}}-\phi_{D}}{\phi_{D}-\phi_{E}}\right) . \\
& D_{d, T}=D_{\phi}+\left(E_{\phi} \frac{\phi_{E}-\phi_{\mathrm{env}}}{\phi_{D}-\phi_{E}}+D_{\phi} \frac{\phi_{\text {core }}-\phi_{D}}{\phi_{D}-\phi_{E}}\right) .
\end{aligned}
$$

Again, the $T$ subscript indicates these $E_{d}$ and $D_{d}$ values have been calculated by transformation of $E_{\phi}$ and $D_{\phi}$, not via Eq. (4). In this case, to convert from $\left(E_{\phi}, D_{\phi}\right)$ to $\left(E_{d, T}, D_{d, T}\right)$, both $E_{\phi}$ and $D_{\phi}$ must be increased by $E_{\phi} a+$ $D_{\phi} b$, where $a=\left(\phi_{E}-\phi_{\text {env }}\right) /\left(\phi_{D}-\phi_{E}\right)$ and $b=\left(\phi_{\text {core }}-\right.$ $\left.\phi_{D}\right) /\left(\phi_{D}-\phi_{E}\right)$.

We now have relationships allowing us to transform the unbiased $E_{d}$ and $D_{d}$ values into biased tracer budget $E_{\phi}$ and $D_{\phi}$ values, which are better suited for simple entraining plume parameterization of cloud fields. Comparison of $E_{q}$ and $D_{q}\left(E_{\phi}\right.$ and $D_{\phi}$ inferred using total specific moisture $q_{t}$ as the tracer) with $E_{d}$ and $D_{d}$ shows that the direct entrainment and detrainment magnitudes are significantly larger than the tracer budget values (Figs. 2b,c, gray and dotted lines). Using Eq. (8) to calculate $E_{q, T}$ and $D_{q, T}$ with $q_{E}=q_{\text {edge }}$, the horizontal mean humidity in the cloud edge, and $q_{D}=q_{\text {shell }}$, the horizontal mean humidity in the cloud shell, results in values quite close to the tracer budget values above the middle of the cloud layer. The transformation also duplicates the negative detrainment values near cloud base that are typically produced by tracer calculations.

\section{b. Preferential entrainment of moist ascending air}

Relative to the tracer budget values, the $E_{q, T}$ and $D_{q, T}$ values calculated using $q_{E}=q_{\text {edge }}$ and $q_{D}=q_{\text {shell }}$ are still too large near cloud base. We can explain this difference as being the result of the mean fluid property values of the entrained and detrained air being different than the mean values of the shell and edge air, respectively. Using the mean shell and edge values of properties to transform the direct entrainment and detrainment assumes that any fluid parcel in the shell or edge is equally likely to be entrained or detrained. In reality, mixing relatively dry air into the cloud core is more likely to cause evaporation, which will drive detrainment, while mixing relatively moist air into the cloud core is more likely to produce a saturated fluid mixture, resulting in entrainment. This suggests that the moistest shell parcels are more likely to undergo entrainment than the average shell parcel, and the driest edge parcels are more likely to detrain than the average edge parcel.

We can directly calculate the effective fluid property values at which entrainment occurs by taking the total fluid property entrainment $(E \phi)_{d}$ [calculated via Eq. (5)] and dividing it by the total mass entrainment $E_{d}$ so that $\phi_{\text {entrain }}=(E \phi)_{d} / E_{d}$. Similarly, the effective property values of the detraining air can be found from 
a) Mean $q_{t}$ Profiles

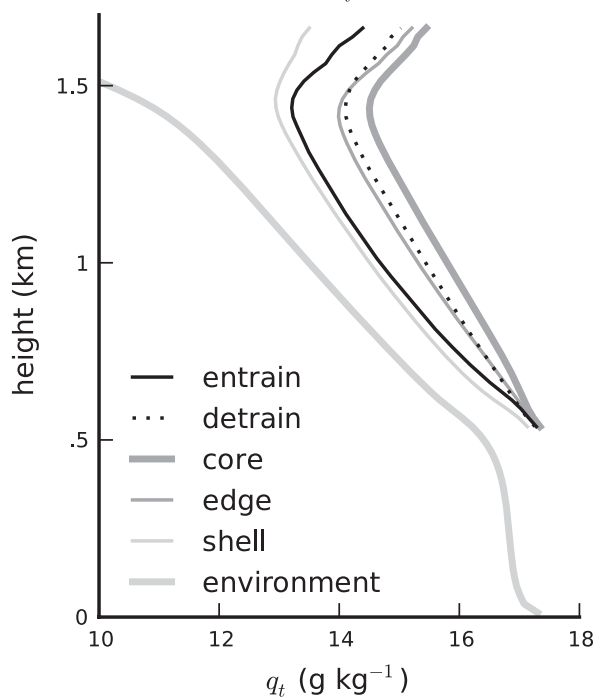

b) Entrainment Values

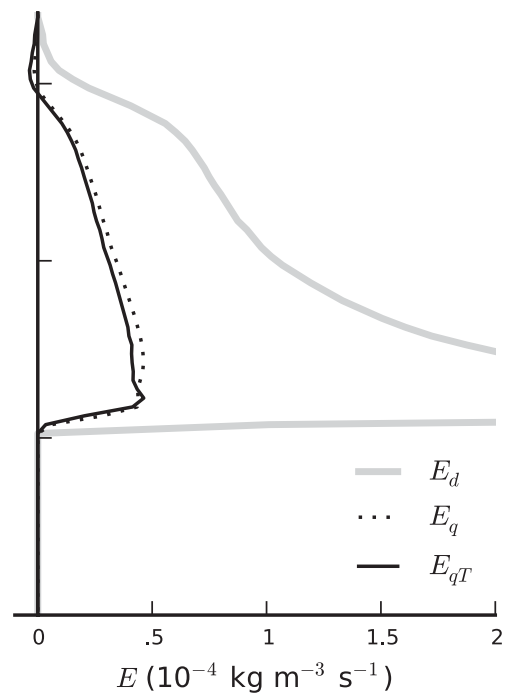

c) Detrainment Values

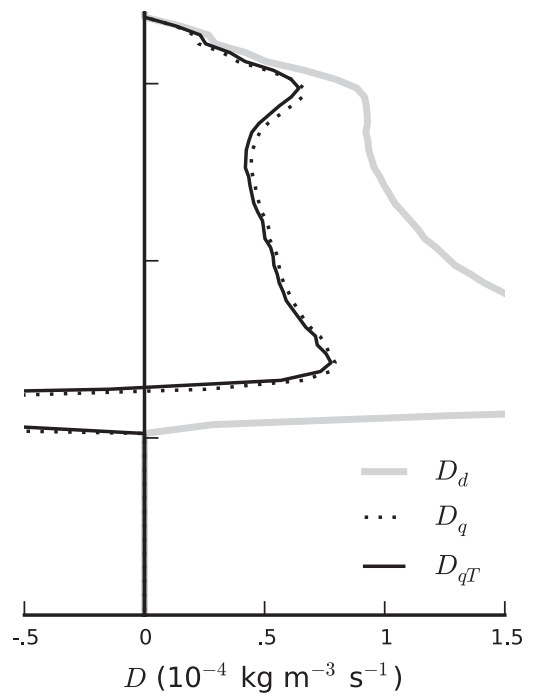

FIG. 3. Result of transforming direct entrainment values into equivalent tracer budget values using effective entrainment and detrainment properties from the BOMEX simulation. (a) Mean profiles of $q_{\text {entrain }}$ (black line), and $q_{\text {detrain }}$ (dotted line), overlaid on the mean total specific water values of the core, edge, shell, and environment. These $q_{t}$ values are used to transform directly calculated values of (b) entrainment and (c) detrainment (gray line) into equivalent tracer budget values (black line). The Siebesma tracer budget entrainment and detrainment are shown for comparison (dotted lines).

$\phi_{\text {detrain }}=(D \phi)_{d} / D_{d}$. Examination of these values from the BOMEX simulation using $q_{t}$ for $\phi$ shows that $q_{\text {entrain }}$ is moister than $q_{\text {shell }}$ (Fig. 3a), indicating that entrainment occurs preferentially at the moistest parts of the shell. Conversely, there is little difference between $q_{\text {detrain }}$ and $q_{\text {edge }}$, indicating that the detrained parcels tend to have the mean moisture of the cloud core edge. Using $q_{\text {entrain }}$ and $q_{\text {detrain }}$ to transform $E_{d}$ and $D_{d}$ reduces the large entrainment and detrainment values near cloud base found using the mean shell and edge properties (solid black line, Figs. $3 \mathrm{~b}, \mathrm{c}$ ), bringing $E_{q, T}$ and $D_{q, T}$ into agreement with $E_{q}$ and $D_{q}$.

\section{4. $E_{q}, E_{h}$, and $E_{w}$ differences}

Equation (8a) or (8b) implies that the tracer budget method will measure different entrainment and detrainment values for fluid properties with differing values of $A=\left(\phi_{E}-\phi_{\text {env }}\right) /\left(\phi_{\text {core }}-\phi_{\text {env }}\right)$ and $B=\left(\phi_{\text {core }}-\phi_{D}\right) /$ $\left(\phi_{\text {core }}-\phi_{\text {env }}\right)$, respectively. Supporting this, Romps (2010, his Fig. 2) showed that changing the sources and sinks applied to an artificial numerical tracer resulted in large changes in measured tracer budget entrainment rates. With this in mind we compare $E_{\phi, T}$ and $D_{\phi, T}$ values produced by $h$ and $w$ with those produced using $q_{t}$.

Liquid water moist static energy shows a similar relative distribution of core, edge, shell, environment, entrained, and detrained properties when compared to $q_{t}$, indicating a tight coupling between these variables in the cloud dynamics. Because these properties are so tightly coupled, $E_{h, T}$ and $D_{h, T}$ values are nearly identical to the $E_{q, T}$ and $D_{q, T}$ (not shown).

Vertical velocity shows very different relative profiles compared to $q_{t}$ or $h$ (cf. Figs. 4a and 3a). There is a much wider spread in the $w$ values, with the shell having nearly zero vertical velocity and the edge being halfway between the core and the environment. The value of $w_{\text {detrain }}$ is slightly larger than the value of $w$ in the cloud core edge, while $w_{\text {entrain }}$ is much larger than $w$ in the shell, becoming roughly the same value as $w_{\text {edge }}$. Since $w_{\text {entrain }}$ and $w_{\text {detrain }}$ are both larger than $w_{\text {shell }}$ and $w_{\text {edge }}$, this implies that rapidly rising air is both preferentially entrained and detrained over slowly rising air. These effective entrainment and detrainment $w$ values produce $E_{w, T}$ and $D_{w, T}$ values (solid black line, Figs. $4 \mathrm{~b}, 4 \mathrm{c}$ ) that are quite different than the entrainment and detrainment produced by $q_{t}$ and $h$ (dotted line, Figs. $4 \mathrm{~b}, \mathrm{c}$ ); $E_{w, T}$ is negative near cloud base while $E_{q, T}$ is positive, and both $E_{w, T}$ and $D_{w, T}$ are half the magnitude of $D_{q, T}$ over much of the cloud layer.

Finally, we examine the temporal variability of $A=$ $\left(\phi_{E}-\phi_{\text {env }}\right) /\left(\phi_{\text {core }}-\phi_{\text {env }}\right)$ and $B=\left(\phi_{\text {core }}-\phi_{D}\right) /\left(\phi_{\text {core }}-\right.$ $\left.\phi_{\text {env }}\right)$ from the transformation Eqs. (8a) and (8b) in the ARM model run. Since $A$ represents the fraction of core air in a mixture of core and environmental air that has the properties of $\phi_{E}$, and $B$ represents the fraction of environmental air in a mixture of core and environmental 


\section{a) Mean $w$ Profiles}

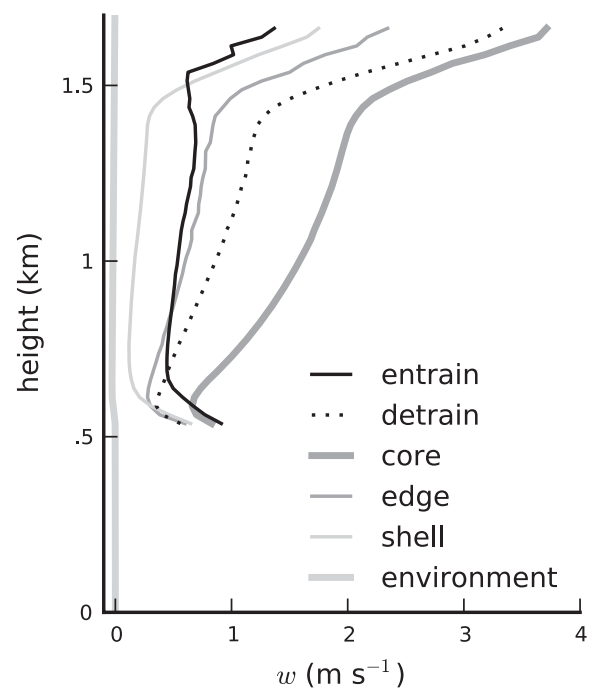

b) Entrainment Values

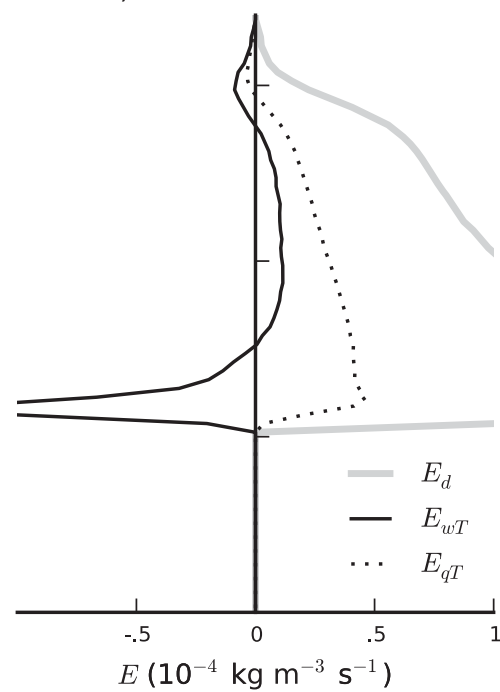

c) Detrainment Values

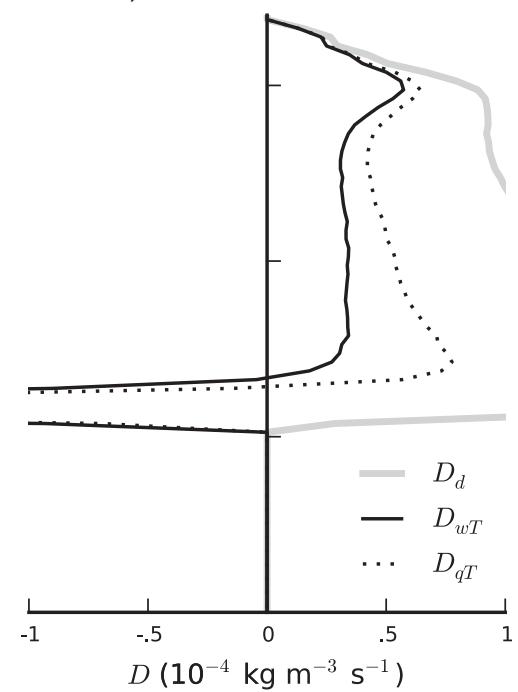

FIG. 4. Result of transforming direct entrainment values from the BOMEX simulation into equivalent $w$ budget values. (a) Mean profiles of the effective $w$ values being entrained (black line), and detrained (dotted line), overlaid on the mean $w$ values of the core, edge, shell, and environment. The entrained and detrained $w$ values are used to transform directly calculated values of (b) entrainment and (c) detrainment (gray line) into equivalent tracer budget values (black line). The entrainment and detrainment values transformed using $q_{t}$ are shown for comparison (dotted lines).

air that has the properties of $\phi_{D}, A$ and $B$ provide information about the reentrainment of previously detrained core fluid and detrainment of previously entrained environmental fluid occurring at different model heights. However, $A$ and $B$ cannot be considered exact mixing fractions, as the source heights of entrained or detrained air mixtures may be different than the height at which they entrain or detrain, and not all mixtures of core and environment properties are equally likely to undergo entrainment.

The values of $A$ and $B$ calculated for $q_{t}$ both show strong changes over the ARM diurnal cycle (Figs. 5a,b). Near cloud base, $A$ is nearly 1 while $B$ is nearly 0 , implying that both the entrained and detrained air have the properties of the cloud core air. This is due to the main entrainment process at cloud base being condensation of rising thermals instead of mixing; buoyant updrafts simply condense without modification of their properties. Similarly, the air that detrains from the clouds at cloud base is almost undiluted because most entrainment at this level comes from the well-mixed subcloud layer below and so has properties nearly identical to the cloud core. At cloud top, $A$ is also nearly 1 while $B$ is nearly 0 , implying that both the entrained and detrained air have the properties of the cloud core air. This is due to cloud core detrainment in the inversion being driven by the cloud becoming negatively buoyant as it enters the steep inversion temperature gradient (Wu et al. 2009). This process is adiabatic, and so the air detraining from the core is undiluted by the environment. Conversely, much of the air surrounding the remaining cloud core that is available for entrainment was previously detrained from the core without mixing, and so has the properties of the core air.

As the clouds detrain into the inversion over the course of the day, they cause the inversion to rise. This means that points in the midcloud layer are less influenced by the adiabatic entrainment and detrainment processes occurring at cloud base and cloud top, and so the effects of mixing become more prominent. By the end of the day, $A$ within the cloud layer has values near 0.6 , suggesting that a significant amount of reentrainment of air previously detrained from the core still occurs. Also, $B$ has values near 0.2 , indicating that air detraining from the core is relatively undiluted by environmental air; this makes sense, since relatively little dilution by environmental air is required to cause the core air to become neutrally buoyant and detrain.

When calculated for $w, A$ reaches a value around 0.4 and $B$ goes to 0.6 near the middle of the cloud layer by the end of the day. The differences between these values and the values of $A$ and $B$ calculated for $q_{t}$ are the result of buoyancy and pressure gradient forces on the mixtures, and the stronger tendency for upward-moving shell parcels to be entrained relative to the tendency to entrain moister shell parcels. In other words, a relatively dry, rapidly ascending shell parcel is more likely to be entrained than a relatively moist, slowly ascending shell parcel. This is especially apparent near cloud base where the values of $A$ are larger than 1, due to the mean entrained 
a) A calculated using $q_{t}$

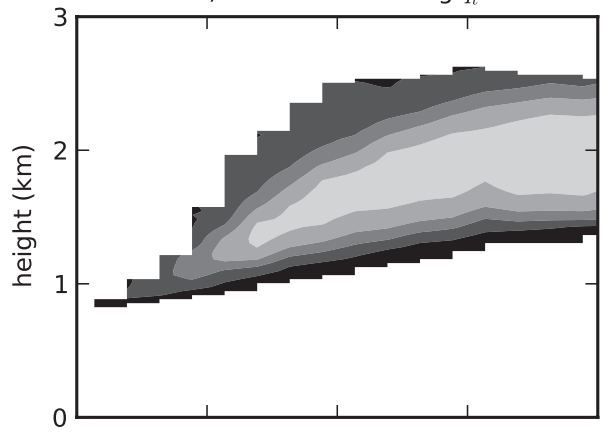

c) A calculated using $w$

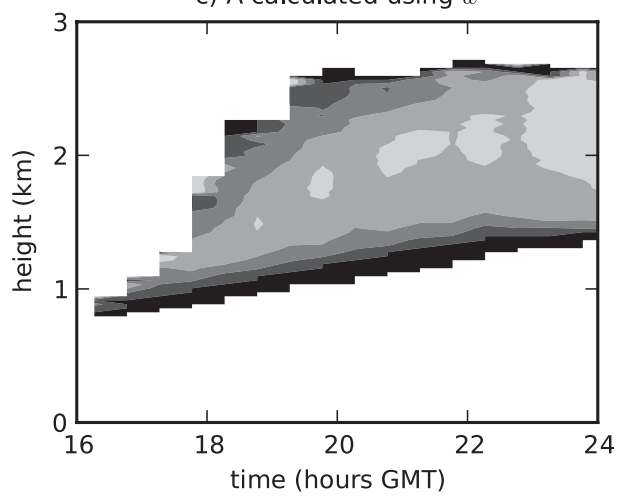

b) B calculated using $q_{t}$

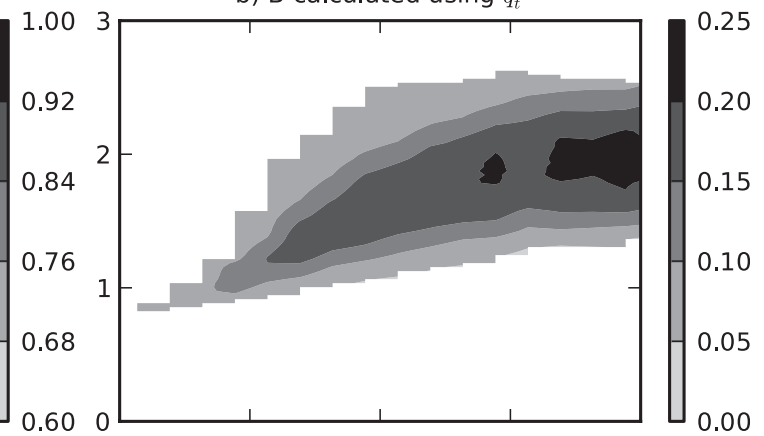

d) B calculated using $w$

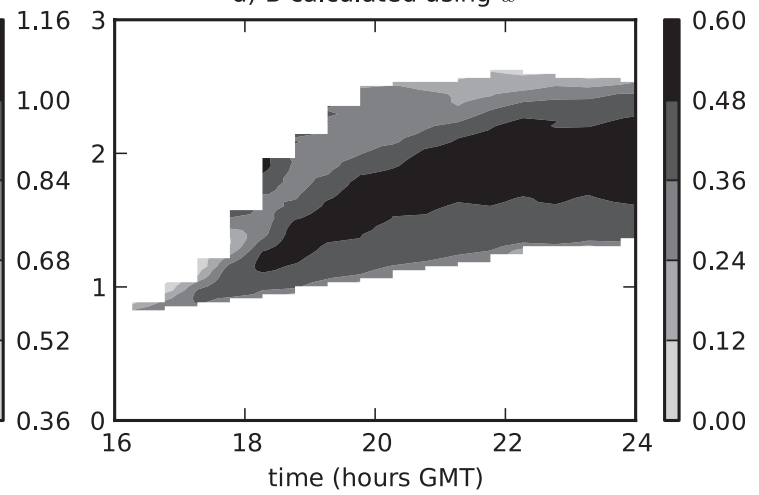

FIG. 5. Variation in (a) the fraction of core air in a mixture of core and environmental air needed to produce the mean humidity entrained by the clouds, (b) the fraction of environmental air in a mixture of core and environmental air needed to produce the mean humidity detrained by the clouds, (c) the fraction of core air in a mixture of core and environmental air needed to produce the mean vertical velocity entrained by the clouds, and (d) the fraction of environmental air in a mixture of core and environmental air needed to produce the mean vertical velocity detrained by the clouds, over the duration of the ARM model run.

parcels having a larger upward velocity than the mean core parcels.

Performing these calculations with fixed values of $\left(\phi_{\text {core }}-\phi_{\text {env }}\right)$, to remove changes due to movement of the mean environment and core profiles, shows similar results. Changes in the properties of the entraining and detraining fluid due to the dynamics of mixing and entrainment in the shell clearly are active in determining the rates at which properties entrain and detrain.

\section{Causes of preferential entrainment of moist ascending air}

The reason that shell air that is moister and ascending faster than the mean shell is more likely to be entrained can be seen by comparing instantaneous snapshots of the model values of local mass entrainment $e$, moisture entrainment $e q_{t}$, and vertical velocity entrainment $e w$. Since the Romps (2010) method of calculating $e$ and $d$ requires taking time averages, it is unsuitable for calculating instantaneous entrainment fields. Instead, we use an alternative method we have devised that substitutes spatial interpolation for time averaging (Dawe and Austin 2011). This alternative method results in slightly smaller values of $e$ and $d$ than those produced by Romps' method, but the two calculations show good agreement in variability. The $e q_{t}$ and $e w$ fields are calculated simply by multiplying the value of $e$ by the values of $q_{t}$ and $w$, respectively.

Comparing the $e, e q_{t}$, and $e w$ fields shows that $e$ and $e q_{t}$ have a very similar spatial pattern, but $e w$ is concentrated in regions where strong updrafts enter the cloud core (Fig. 6). The reason for this can been seen by examining the buoyancy, condensed liquid water, and vertical velocity fields that define the cloud core. Of these three fields, buoyancy is the strongest constraint determining if air is part of the core. However, regions exist far above cloud base where air has become negatively buoyant but maintains upward velocity and condensed liquid water. As this air continues to rise, more condensation occurs, which heats the updraft, makes it positively buoyant, and thus entrains it into the core. In this way, entrainment is positively correlated with both $q_{t}$ and $w$. This process occurs fairly often in our model cloud field, as evidenced both by our manual examination of the 
a) $E\left(10^{-1} \mathrm{~kg} \mathrm{~m}^{-3} \mathrm{~s}^{-1}\right)$

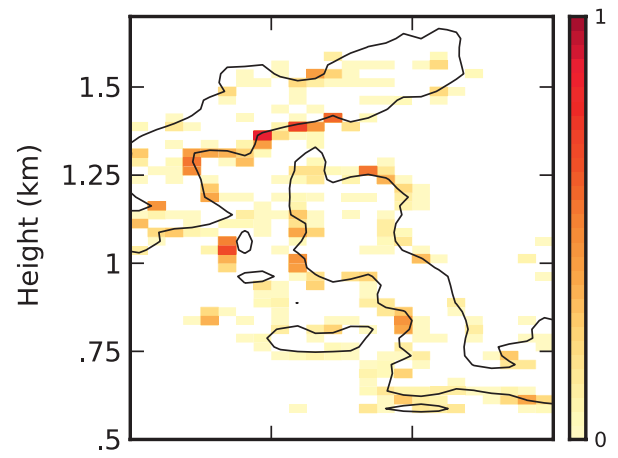

d) $T_{v}-\operatorname{mean}\left(T_{v}\right)(\mathrm{K})$

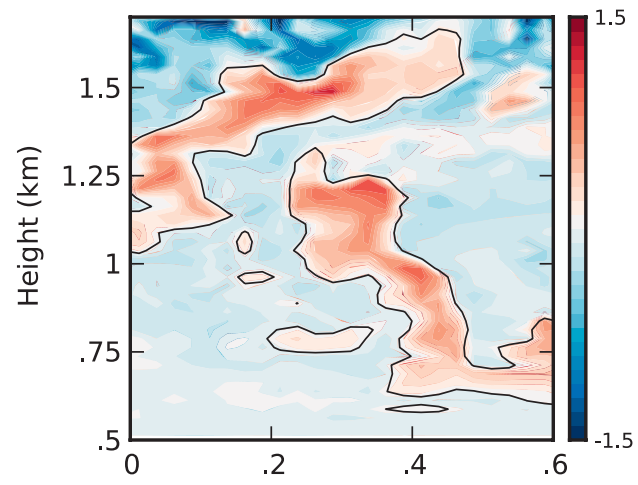

b) $E q_{t}\left(10^{-3} \mathrm{~kg} \mathrm{~m}^{-3} \mathrm{~s}^{-1}\right)$

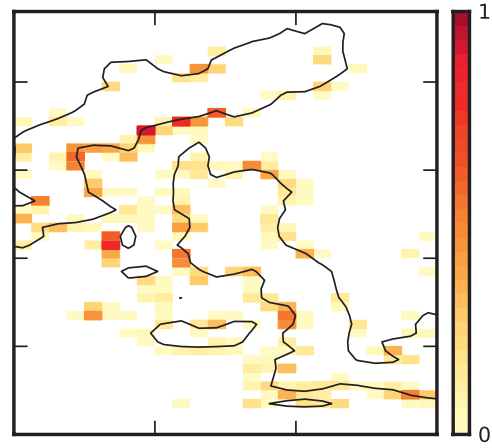

e) $q_{n}\left(\mathrm{~g} \mathrm{~kg}^{-1}\right)$

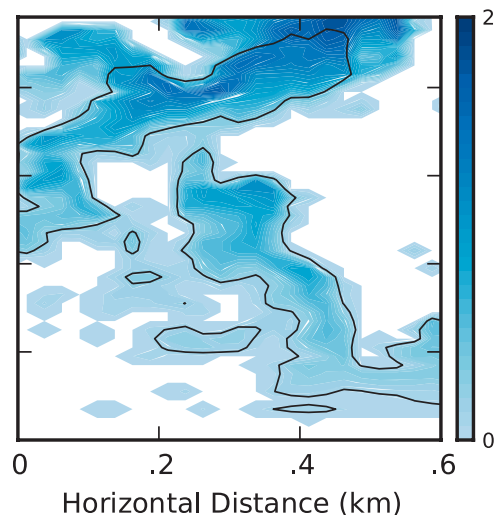

c) $E w\left(10^{-1} \mathrm{~kg} \mathrm{~m}^{-2} \mathrm{~s}^{-2}\right)$

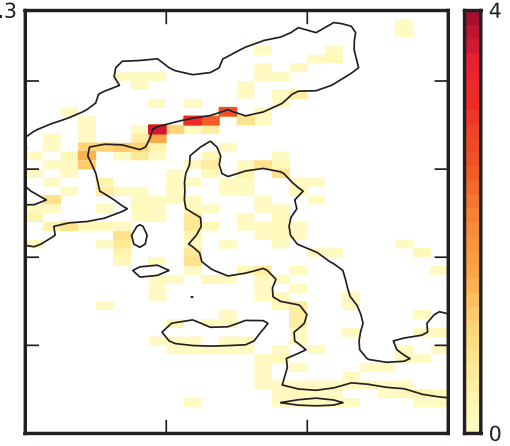

f) $w\left(\mathrm{~m} \mathrm{~s}^{-1}\right)$

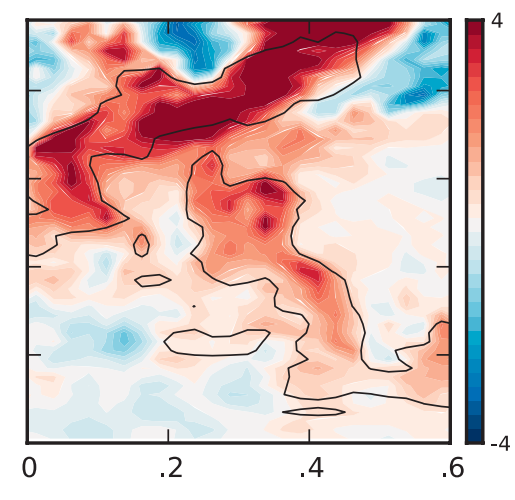

FIG. 6. Instantaneous vertical cross section of (a) directly calculated cloud core mass entrainment, (b) humidity entrainment, (c) vertical velocity entrainment, (d) buoyancy, (e) condensed liquid water, and (f) vertical velocity of a single BOMEX simulation cloud, illustrating the tendency to entrain shell air that is rising faster than the mean shell. Black lines indicate the edge of the cloud core in each figure.

output fields and by the size of the difference between $w_{\text {shell }}$ and $w_{\text {entrain }}$ in the mean profiles.

While the tendency for rapidly ascending shell air to be entrained more often than the slower parts of the shell was found for cloud core entrainment, we would like to emphasize that this process is not an artifact of the cloud core sampling; similar results appear when we perform entrainment calculations for simple cloudy regions (areas of condensed liquid water). In this case, vertical advection of air can drive condensation, converting environment air into cloud air and thus driving entrainment of air into the cloud.

\section{Discussion}

Considering all these results, we now turn to the most important question of all: which entrainment value is the right one? The unsatisfying answer is that it depends on the purpose for which the entrainment is to be used.

Consider a cumulus cloud plume model based on a simplified form of the continuity equation that assumes the cloud fraction is constant,

$$
\frac{\partial M_{\text {core }}}{\partial z}=E-D
$$

a cloud budget equation for a conserved variable $\phi$ that assumes mean vertical advection is balanced by entrainment of mean environmental properties,

$$
M_{\text {core }} \frac{\partial \phi_{\text {core }}}{\partial z}=E\left(\phi_{\text {env }}-\phi_{\text {core }}\right)
$$

and a simple detrainment forcing equation,

$$
\rho \frac{\partial \phi_{\text {env }}}{\partial t}=D\left(\phi_{\text {core }}-\phi_{\text {env }}\right)
$$

The term $\phi_{\text {env }}$ is input to the parameterization from the GCM. If we assume we have a perfect parameterization of $M_{\text {core }}$ and $\phi_{\text {core }}$ at cloud base with which to construct mean core mass flux and tracer profiles, we wish the $E$ and $D$ values to produce a profile of $\partial \phi_{\mathrm{env}} / \partial t$ to force the GCM that agrees with LES results for a similar mean environmental profile. The $E$ and $D$ we desire then are 
closer to $E_{\phi}$ and $D_{\phi}$ than to $E_{d}$ and $D_{d}$ but nevertheless are not identical to $E_{\phi}$ and $D_{\phi}$ because of neglect of the time tendency and Reynolds flux budget terms in Eqs. (11) and (12). This also implies that we should have different $E_{\phi}$ and $D_{\phi}$ values for properties with different distribution patterns around the clouds.

Using values near $E_{d}$ and $D_{d}$ instead would require modifying Eq. (11) to

$$
M_{\text {core }} \frac{\partial \phi_{\text {core }}}{\partial z}=E\left(\phi_{\text {core }}-\phi_{E}\right)-D\left(\phi_{\text {core }}-\phi_{D}\right)
$$

and Eq. (12) to

$$
\rho \frac{\partial \phi_{\mathrm{env}}}{\partial t}=D\left(\phi_{D}-\phi_{\mathrm{env}}\right)-E\left(\phi_{E}-\phi_{\mathrm{env}}\right) .
$$

Now, instead of calculating different $E$ and $D$ values for each tracer we wish to model, we must instead calculate $\phi_{E}$ and $\phi_{D}$ values for each property that is entrained or detrained. While it is possible this would produce a better parameterization, it seems simpler to fold the effects of $\phi_{E}$ and $\phi_{D}$ into the $E$ and $D$ values and keep the equations in their less complex forms. On the other hand, the true values of the mass entrainment and detrainment are important for comparison of LES results with field studies using Doppler radar to estimate entrainment and detrainment velocities, or possibly for calculations of aerosol reactions whose chemical properties are dependent on the concentration of liquid water in the air (Hoppel et al. 1994). They are also vital for diagnosing mass exchanges of individual clouds in an LES ensemble, for which a simple "environment" and "cloud core" mean tracer budget may be difficult to define.

The large positive values of $w_{\text {entrain }}$ are clearly inconsistent with the often-made assumption that fluid entrained into the cloud has negligible vertical momentum (Siebesma et al. 2003). This is reflected in the smaller values of the transformed $E_{w, T}$ and $D_{w, T}$ shown in Fig. 4. The negative values of $E_{w}, E_{q}$, and $D_{w}$ produced near cloud base emphasize the artificial nature of the tracer budget entrainment and detrainment. Rather, $E_{\phi}$ and $D_{\phi}$ should be interpreted simply as mathematical quantities that satisfy both the continuity Eq. (2) and the tracer budget of the cloud core under the assumption that the core entrains mean environment fluid and detrains mean cloud core fluid.

As both BOMEX and ARM model simulations involved nonprecipitating shallow cumulus, we have ignored the effects of precipitation. Precipitation is generally not considered part of the turbulent mixing processes associated with entrainment and detrainment in parameterization, instead being represented by a sink term in the liquid water budget (Tiedtke 1989; Kain and
Fritsch 1990). Nevertheless, incorporating precipitation into the tracer budget and direct entrainment calculations would be relatively simple. The precipitation flux divergence would be a new sink/source forcing term in Siebesma's Eq. (3), and would be part of the forcing term $\rho \mathcal{A} S_{\phi}$ in Romps' Eq. (5), resulting in precipitation flux divergence not being counted as part of the detrainment. Specifying the advection terms would be somewhat trickier since, depending on the complexity of the microphysics scheme, moisture might be advected as a single $q_{t}$ field or advected as separate hydrometeor classes. However, this would simply mean adding extra advection terms for each hydrometeor class. Once these effects were properly incorporated into the calculations, the transformations between $\left(E_{d}, D_{d}\right)$ and $\left(E_{q}, D_{q}\right)$ would be unchanged.

\section{Conclusions}

We have explained the differences between values of cloud core entrainment and detrainment of mass calculated via tracer budget and direct calculations as the result of the presence of a shell of moist air around the cloud cores and drier air at the edge of the cloud core, and the tendency for the mean tracer value of the entrained fluid to be greater than the mean tracer value of the cloud shell. Relaxing the assumption made by Siebesma and Cuijpers (1995) that entrained and detrained fluid has the properties of the environment and core, respectively, allows us to transform direct entrainment/detrainment values into corresponding tracer budget values suitable for use in simple entraining plume parameterizations of cumulus convection.

The moistest, fastest-rising regions of the shell are entrained more often than drier, slower-rising regions. This tendency results from thermodynamic phase changes in rising air parcels. Condensation in upward-moving, saturated, but negatively buoyant air parcels causes latent heating, increasing the air's buoyancy and causing it to entrain into the core. This effect suggests that the dynamics of the moist cloud shell have a role in mediating fluxes between the clouds and the environment.

Transforming directly calculated values of entrainment and detrainment into tracer budget values results in different entrainment and detrainment values for total specific water $q_{t}$ and liquid-water moist static energy $h$ than for vertical velocity $w$. Furthermore, the tracer budget values of entrainment and detrainment compatible with $q_{t}, h$, and $w$ exhibit different patterns of variability over the course of a diurnal cycle in a GCSS ARM LES. This suggests that cumulus cloud parameterizations based on bulk models should use different $E$ and $D$ values for $q_{t}$ and $h$ than for $w$, and it raises the possibility that different 
TABLE A1. List of symbols.

\begin{tabular}{|c|c|c|c|}
\hline Symbol & Unit & Definition & First occurrence \\
\hline$E(z), D(z)$ & $\mathrm{kg} \mathrm{m}^{-3} \mathrm{~s}^{-1}$ & Cloud core mass entrainment, detrainment rate & Eq. (2) \\
\hline$E_{\phi}(z), D_{\phi}(z)$ & $\mathrm{kg} \mathrm{m}^{-3} \mathrm{~s}^{-1}$ & $\begin{array}{l}\text { Mass entrainment, detrainment rate calculated } \\
\text { using the Siebesma tracer budget }\end{array}$ & Eqs. (3a), (3b) \\
\hline$e(x, y, z), d(x, y, z)$ & $\mathrm{kg} \mathrm{m}^{-3} \mathrm{~s}^{-1}$ & $\begin{array}{l}\text { Local mass entrainment, detrainment rate calculated } \\
\text { directly from model velocity and tracer fields }\end{array}$ & Eq. (4) \\
\hline$E_{d}(z), D_{d}(z)$ & $\mathrm{kg} \mathrm{m}^{-3} \mathrm{~s}^{-1}$ & $\begin{array}{l}\text { Cloud core mass, detrainment rate calculated from the } \\
\text { horizontal sum of } e(x, y, z) \text { or } d(x, y, z)\end{array}$ & Section 1 \\
\hline$(e \phi)(x, y, z),(d \phi)(x, y, z)$ & {$[\phi] \mathrm{kg} \mathrm{m}^{-3} \mathrm{~s}^{-1}$} & $\begin{array}{l}\text { Local cloud core } \phi \text { entrainment, detrainment rate calculated } \\
\text { directly from model velocity and tracer fields }\end{array}$ & Eq. (5) \\
\hline$(E \phi)_{d}(z),(D \phi)_{d}(z)$ & {$[\phi] \mathrm{kg} \mathrm{m}^{-3} \mathrm{~s}^{-1}$} & $\begin{array}{l}\text { Cloud core } \phi \text { entrainment, detrainment rate calculated from the } \\
\text { horizontal sum of }(e \phi)(x, y, z) \text { or }(d \phi)(x, y, z)\end{array}$ & Section 2 \\
\hline$E_{\phi, T}(z), D_{\phi, T}(z)$ & $\mathrm{kg} \mathrm{m}^{-3} \mathrm{~s}^{-1}$ & $\begin{array}{l}\text { Mass entrainment, detrainment rate calculated by transforming } \\
\text { direct values into budget values }\end{array}$ & Eqs. (8a), (8b) \\
\hline$E_{d, T}(z), D_{d, T}(z)$ & $\mathrm{kg} \mathrm{m}^{-3} \mathrm{~s}^{-1}$ & $\begin{array}{l}\text { Cloud core mass entrainment, detrainment rate calculated by } \\
\text { transforming tracer budget values into direct values }\end{array}$ & Section 1 \\
\hline$\phi(x, y, z)$ & {$[\phi]$} & Any fluid property, such as $q_{t}\left(\mathrm{~kg} \mathrm{~kg}^{-1}\right), h\left(\mathrm{~J} \mathrm{~kg}^{-1}\right)$, or $w\left(\mathrm{~m} \mathrm{~s}^{-1}\right)$ & Section 1 \\
\hline$\phi_{\text {core }}(z)$ & {$[\phi]$} & Mean cloud core $\phi$ & Section $3 \mathrm{a}$ \\
\hline$\phi_{\text {edge }}(z)$ & {$[\phi]$} & Mean cloud edge $\phi$ & Section $3 \mathrm{a}$ \\
\hline$\phi_{\text {shell }}(z)$ & {$[\phi]$} & Mean cloud shell $\phi$ & Section $3 \mathrm{a}$ \\
\hline$\phi_{\mathrm{env}}(z)$ & {$[\phi]$} & Mean environment $\phi$ & Section $3 \mathrm{a}$ \\
\hline$\phi_{\text {entrain }}(z)$ & {$[\phi]$} & Effective value of $\phi$ being entrained calculated from $(E \phi)_{d} / E_{d}$ & Section $3 b$ \\
\hline$\phi_{\text {detrain }}(z)$ & {$[\phi]$} & Effective value of $\phi$ being detrained calculated from $(D \phi)_{d} / D_{d}$ & Section $3 b$ \\
\hline$\phi_{E}(z)$ & {$[\phi]$} & Placeholder for the value of $\phi$ assumed to be entraining & Eq. (8a) \\
\hline$\phi_{D}(z)$ & {$[\phi]$} & Placeholder for the value of $\phi$ assumed to be detraining & Eq. (8b) \\
\hline
\end{tabular}

entrainment and detrainment rates apply to other cloud properties, such as aerosol concentrations.

Direct entrainment and detrainment calculations are powerful tools that should be used to help improve our understanding of the dynamics of mass exchanges between clouds and their environment, with an eye to folding these effects into the simplest parameterizations possible. Doing so has the potential to improve GCM parameterization of the magnitude and variability of mass and tracer exchanges between clouds and their environment.

Acknowledgments. Support for this work was provided by the Canadian Foundation for Climate and Atmospheric Science through the Cloud Aerosol Feedback and Climate network. We thank Marat Khairoutdinov for making SAM available to the cloud modeling community. We would also like to thank David Romps and two anonymous reviewers whose comments significantly improved the quality of this paper. All figures were generated using the matplotlib library in the Python programming language.

\section{APPENDIX}

\section{Table of Notation}

Table A1 shows the variables, along with their definitions and units, used in this study.

\section{REFERENCES}

Bechtold, P., M. Köhler, T. Jung, F. Doblas-Reyes, M. Leutbecher, M. J. Rodwell, F. Vitart, and G. Balsamo, 2008: Advances in simulating atmospheric variability with the ECMWF model: From synoptic to decadal time-scales. Quart. J. Roy. Meteor. Soc., 134, 1337-1351, doi:10.1002/qj.289.

Brown, A. R., and Coauthors, 2002: Large-eddy simulation of the diurnal cycle of shallow cumulus convection over land. Quart. J. Roy. Meteor. Soc., 128, 1075-1093.

Dawe, J. T., and P. H. Austin, 2011: Interpolation of LES cloud surfaces for use in direct calculations of entrainment and detrainment. Mon. Wea. Rev., 139, 444-456.

de Rooy, W. C., and A. P. Siebesma, 2010: Analytical expressions for entrainment and detrainment in cumulus convection. Quart. J. Roy. Meteor. Soc., 136, 1216-1227, doi:10.1002/qj.640.

Heus, T., and H. J. J. Jonker, 2008: Subsiding shells around shallow cumulus clouds. J. Atmos. Sci., 65, 1003-1018.

—, C. F. J. Pols, H. J. J. Jonker, H. E. A. Van den Akker, and D. H. Lenschow, 2009: Observational validation of the compensating mass flux through the shell around cumulus clouds. Quart. J. Roy. Meteor. Soc., 135, 101-112.

Hoppel, W. A., G. M. Frick, J. Fitzgerald, and R. E. Larson, 1994: Marine boundary-layer measurements of new particle formation and the effects nonprecipitating clouds have on aerosol size distribution. J. Geophys. Res., 99, 14 443-14 459.

Jonas, P. R., 1990: Observations of cumulus cloud entrainment. Atmos. Res., 25, 105-127.

Jonker, H. J. J., T. Heus, and P. P. Sullivan, 2008: A refined view of vertical mass transport by cumulus convection. Geophys. Res. Lett., 35, L07810, doi:10.1029/2007GL032606.

Kain, J. S., and J. M. Fritsch, 1990: A one-dimensional entraining/ detraining plume model and its application in convective parameterization. J. Atmos. Sci., 47, 2784-2802. 
Khairoutdinov, M. F., and D. A. Randall, 2003: Cloud resolving modeling of the ARM summer 1997 IOP: Model formulation, results, uncertainties, and sensitivities. J. Atmos. Sci., 60, 607-625.

Randall, D., and Coauthors, 2003: Confronting models with data: The GEWEX Cloud Systems Study. Bull. Amer. Meteor. Soc., 84, 455-469.

Rodts, S. M. A., P. G. Duynkerke, and H. J. J. Jonker, 2003: Size distributions and dynamical properties of shallow cumulus clouds from aircraft observations and satellite data. J. Atmos. Sci., 60, 1895-1912.

Romps, D. M., 2010: A direct measure of entrainment. J. Atmos. Sci., 67, 1908-1927.

Rougier, J., D. M. H. Sexton, J. M. Murphy, and D. Stainforth, 2009: Analyzing the climate sensitivity of the HadSM3 climate model using ensembles from different but related experiments. J. Climate, 22, 3540-3557.

Siebesma, A. P., 1998: Shallow cumulus convection. Buoyant Convection in Geophysical Flows, E. J. Plate, Ed., Kluwer Academic, 441-486.
_ , and J. W. M. Cuijpers, 1995: Evaluation of parametric assumptions for shallow cumulus convection. J. Atmos. Sci., 52, 650-666.

- and Coauthors, 2003: A large-eddy simulation intercomparison study of shallow cumulus convection. J. Atmos. Sci., 60, 12011219.

Stainforth, D. A., and Coauthors, 2005: Uncertainty in predictions of the climate response to rising levels of greenhouse gases. Nature, 433, 403-406.

Tiedtke, M., 1989: A comprehensive mass flux scheme for cumulus parameterization in large-scale models. Mon. Wea. Rev., 117, 1779-1800.

Wang, Y., and B. Geerts, 2010: Humidity variations across the edge of trade wind cumuli: Observations and dynamical implications. Atmos. Res., 97, 144-156, doi:10.1016/j.atmosres.2010. 03.017.

Wu, C.-M., B. Stevens, and A. Arakawa, 2009: What controls the transition from shallow to deep convection? J. Atmos. Sci., 66, 1793-1806. 